Article

\title{
Explanation of the Patterns, Spatial Relationships, and Node Functions of Biodiversity and Island: An Example of Nature Reserves in Guizhou, Southwest China
}

\author{
Lingbin Yan ${ }^{1} \mathbb{C}$, Lifei $\mathrm{Yu}^{1,2, *} \mathbb{C}$, Mingtai An ${ }^{1,2,3, *}$, Haijun $\mathrm{Su}^{1,2,3}, \mathrm{He} \mathrm{Li}^{4}$ and Congjun Yuan ${ }^{4}$ \\ 1 The Key Laboratory of Plant Resources Conservation and Germplasm Innovation in Mountainous \\ Region (Ministry of Education)/Collaborative Innovation Center for Mountain Ecology and \\ Agro-Bioengineering (CICEAB), College of Life Sciences/Institute of Agro-Bioengineering, \\ Guizhou University, Guiyang 550025, China; link_yan@163.com (L.Y.); haijun_su@163.com (H.S.) \\ 2 Research Center of Karst Ecological Environment, Guizhou University, Guiyang 550025, China \\ 3 Research Center for Biodiversity and Nature Reserve, Guizhou University, Guiyang 550025, China \\ 4 Guizhou Academy of Forestry, Guiyang 550011, China; gdlihe0812@163.com (H.L.); \\ ycongjun2012gzdx@163.com (C.Y.) \\ * $\quad$ Correspondence: lfyu@gzu.edu.cn (L.Y.); gdanmingtai@126.com (M.A.); Tel.: +86-0851-88420800 (L.Y.)
}

Received: 28 September 2019; Accepted: 4 November 2019; Published: 6 November 2019

\begin{abstract}
Biological habitat islanding occurs with the expansion of human activities. Nature reserves are biodiversity hotspots and sources of biodiversity diffusion. To explore the geographical causes of biodiversity and the impact of habitat island on biodiversity, we studied the spatial network relationships of biodiversity in nature reserves and the spatial characteristics of ecological corridors in reserves using various biodiversity indicators and ecological factors of important nature reserves, digital elevation models, and information regarding the land use types in Guizhou Province. Data were analyzed using canonical correspondence analysis and the lowest-cost analysis method. The results of this study showed that the factors that determine the biodiversity of the dominant region are heat, moisture, rock type, parent rock, and soil type. The nature reserves can be divided into seven categories according to the characteristics and ecological factors of the biodiversity network. We identified ecological corridors for biodiversity diffusion and classified them by levels of importance according to their degree of corridor composition.
\end{abstract}

Keywords: biodiversity spatial network; ecological corridor; nature reserve; Guizhou

\section{Introduction}

The expansion of the range and increase in the intensity of human activities have resulted in the fragmentation of the habitats of many organisms [1,2]. Fragmentation effects have also exacerbated the isolation of species, changed natural ecological patterns and processes [3], and contributed to biodiversity loss. Biodiversity loss [4] is a major challenge [5,6] for the natural ecosystems throughout the world. Therefore, the processes, mechanisms, impacts, and solutions related to islanding have been popular topics of biodiversity research $[7,8]$. However, in the fields of biodiversity and conservation, the current research focuses on the impacts of fragmentation on biodiversity $[9,10]$.

Nature reserves commonly serve as islands of biodiversity and a source of biodiversity diffusion. However, the isolation and encirclement caused by human activities, such as transportation, construction, and urban expansion, result in the formation of habitat islands, making material and energy circulation difficult. These islands have a negative effect on biodiversity $[3,10]$. 
To solve these problems, landscape ecology studies have focused on constructing ecological corridors to connect fragmented habitats, increase biological communication between different habitats, mitigate the impact of reproductive isolation, form pathways for biological migration and transmission, and create a stable biodiversity network. However, domestic research and practices are rare [11,12].

In order to study the main factors and influencing factors of biodiversity, understand the characteristics and categories of biodiversity in regional nature reserves, and provide support for improving the conservation efficiency of regional biodiversity, as of 2018, 11 national-level (contains 1 rare fish reserve) and 12 provincial-level nature reserves had been built in Guizhou Province, China, with a total area of $38.99 \mathrm{~km}^{2}$. Although these reserves are diverse in terms of reserve type and the species within them, the nature reserves are scattered, and the islanding phenomenon is obvious. Therefore, 22 nature reserves that protect terrestrial organisms or ecosystems (excluding 1 rare fish reserve) that have been selected to be promoted to the provincial level were selected for evaluation in this study. Canonical correspondence analysis (CCA) was used to analyze the relationship and classification characteristics among ecological factors, regional biodiversity, and nature reserves. Based on the spatial distance, barrier factors, and habitat factors of nature reserves, the resistance surface model of biodiversity was constructed, and the minimum cost path method was used to analyze the diffusion potential path of biodiversity. The results related to macroregional biodiversity characteristics and their relationships with ecological factors will also have a clear supporting role in the study of nature reserves and may lead to the exploration of ecological protection and the construction of ecological corridors in Guizhou Province.

\section{Materials and Methods}

\subsection{Materials}

Data on the members of several species, genera, and families of gymnosperms and angiosperms were collected, such as number of total species (T1), number of seed plants species (P2), number of seed plant genera (P3), number of seed plant families (P4), number of gymnosperm species (P5), number of gymnosperm genera (P6), number of gymnosperm families (P7), number of angiosperm species (P8), number of angiosperm genera (P9), number of angiosperm families (P10), number of spore plant species (P25), number of spore plant genera (P26), and number of spore plant families (P27). It included those of relatively diverse taxa, such as number of Rosaceae species (P11), number of Rosaceae genera (P12), number of Compositae species (P13), number of Compositae genera (P14), number of Gramineae species (P15), number of Gramineae genera (P16), number of Orchidaceae species (P17), number of Orchidaceae families (P18), number of Labiatae species (P19), and number of Labiatae genera (P20); and included those of a relatively old origins, such as number of Polygonaceae species (P21), number of Polygonaceae genera (P22), number of Magnoliaceae species (P23), number of Magnoliaceae families (P24), number of Hepaticae species (P28), number of Hepaticae genera (P29), number of Hepaticae families (P30), number of moss species (P31), number of moss genera (P32), number of moss families (P33), number of fern species (P34), number of fern genera (P35), and number of fern families (P36). Animal information on the members of species, families, and orders of Mammalia, Aves, Reptilia, Amphibia, Pieces, and Insecta was also collected, including number of animal species (A37), number of mammal species (A38), number of mammal species (A39), number of orders (A40), number of Aves species (A41), number of Aves families (A42), number of Aves orders (A43), number of reptile species (A44), number of reptile families (A45), number of reptile orders (A46), number of amphibian species (A47), number of amphibian families (A48), number of amphibian orders (A49), number of fish species (A50), number of fish families (A51), number of fish orders (A52), number of insect species (A53), number of insect families (A54), and number of insect orders (A55). Vegetation subtype formations, number of grassland type (V56), number of shrubs type (V57), number of bamboo forest type (V58), number of evergreen broad-leaved forest type (V59), number of evergreen deciduous broad-leaved mixed forests type (V60), number of deciduous broad-leaved forest type (V61), number of mossy 
dwarf forests type (V62), number of shrub forests type (V63), number of coniferous and broad-leaved mixed forest type (V64), number of coniferous forest type (V65), number of economic forest type (V66), number of agricultural vegetation type (V67), number of moist meadow type (V68), number of aquatic vegetation type (V69), and number of swamp type (V70) were used as regional biodiversity indicators. The altitudinal range, average temperature, average precipitation, plant geographical composition which included the proportion of world distribution, pantropical distribution, distribution of tropical Asia and tropical America, tropical distribution in the old world, distribution from tropical Asia to tropical Oceania, distribution of tropical Asia to tropical Africa, distribution of tropical Asia (India-Malaysia), north temperate zone distribution, discontinuous distribution in East Asia and North America, temperate distribution in the old world, temperate Asian distribution, distribution of the Mediterranean, West Asia and Central Asia, Central Asia distribution, East Asian distribution, endemic distribution in China, number of soil subclass, and number of lithology were used as regional environmental factors. Nature reserves boundary vector data, Guizhou Province land use data (2010, $90 \mathrm{~m}$ resolution, provided by http://westdc.westgis.ac.cn), and SRTM3 digital elevation model (DEM) (V4.1) data (90 m resolution, provided by http://www.gscloud.cn) were used as GIS spatial data.

\subsection{Analysis Methods}

After standardizing the biodiversity data according to the range of indicator values, we drew a heat map, calculated the Euclidean distance between the indicators and used the average distance method for clustering, used the Pearson method to calculate the correlation coefficient between the indicators, selected the correlation coefficient $\geq 0.6$ and the $p$ value $<0.01$ to draw a network diagram based on standardized data, and analyzed the characteristics and modularity of network relationships. To understand the impact factors of biodiversity, the constrained ranking method, the canonical correspondence analysis (CCA), was used for biodiversity data analysis to establish an environmental interpretation model. We performed 9999-times permutation tests on the model, selected the optimal environmental interpretation model, and plotted the corresponding sort map. The principle of CCA refers to Numerical Ecology [13]; the calculation process refers to Numerical Ecology with R [14].

Based on the biological preference for habitat selection, and the size of the terrain to the biological diffusion barrier, combined with the spatial location of the nature reserve, we established a resistance surface model for biodiversity diffusion. The reasons and methods of assignment are described in detail below. Potential ecological corridors, the easiest path for biological spread, are calculated using the least-cost path module between the nature reserves.

\subsubsection{Habitat Selection Preference}

The spread of biodiversity is affected by differences in taxa and different methods of transmission and is influenced at the species level by variations in environmental preferences. Under almost the same conditions in the natural environment (excluding human interference), we assumed that biodiversity will show increase scale, biological enrichment, and diffusion, and a region with relatively high biodiversity and a certain width will objectively form a continuous ecosystem and species habitat as a biodiversity diffusion corridor. Based on this, the land use data (2010) from Guizhou Province (Southwest Mountain point-source data, National Earth System Science Data Sharing Infrastructure, National Science and Technology Infrastructure of China, http://www.geodata.cn) were used as the basic data. Moreover, the degrees of biodiversity associated with the various land use types and the statuses of human activity interference were considered, and different biodiversity diffusion resistance values were calculated (Table 1). 
Table 1. Resistance to biodiversity transmission according to land use type.

\begin{tabular}{lccccc}
\hline Code & Land Use Type & $\begin{array}{c}\text { Biodiversity } \\
\text { Diffusion } \\
\text { Resistance }\end{array}$ & Code & Land Use Type & $\begin{array}{c}\text { Biodiversity } \\
\text { Diffusion } \\
\text { Resistance }\end{array}$ \\
\hline 21 & Woodland & 0 & 52 & Rural settlement & 100 \\
22 & Shrubbery & 10 & 53 & Industrial and mining land & 90 \\
23 & Sparse woodland & 5 & 62 & Bare soil & 30 \\
24 & Operating land or economic woodland & 10 & 64 & Marsh wetland & 40 \\
31 & High-coverage grass & 20 & 66 & Bare rock & 100 \\
32 & Middle-coverage grass & 25 & 111 & Mountain paddy field & 50 \\
33 & Low-coverage grass & 30 & 112 & Hilly paddy field & 60 \\
41 & River & 70 & 113 & Pingba paddy field & 70 \\
42 & Lake & 90 & 114 & Steep slope paddy field & 40 \\
43 & Reservoir & 90 & 121 & Mountain dry land & 50 \\
44 & Snow cover & 100 & 122 & Hilly dry land & 60 \\
46 & Floodplain & 100 & 123 & Pingba dry land & 70 \\
51 & Urban land & 124 & Steep slope dry land & 40 \\
\hline
\end{tabular}

\subsubsection{Geographical Barriers}

In addition to the different types of barriers/connections, the spread of biodiversity is also blocked and indirectly affected by surface morphology. Even though animals have different living habits, most animals are inclined to select a relatively flat habitat from the perspective of energy dissipation. For plants, where the slope is smoother, there is usually a deeper soil layer, which provides high levels of nutrients and water. Plants are more likely to inhabit the least stressful locations.

Therefore, in this study, a slope elevation grid was constructed using the Guizhou DEM, and the different slopes were assigned according to Table 2.

Table 2. Resistance of geographical barriers.

\begin{tabular}{ccccccccc}
\hline No. & Slope $/{ }^{\circ}$ & Resistance & No. & Slope $/{ }^{\circ}$ & Resistance & No. & Slope $/{ }^{\circ}$ & Resistance \\
\hline 1 & $0 \sim 5$ & 0 & 3 & $15 \sim 25$ & 15 & 5 & $35 \sim 70$ & 50 \\
2 & $5 \sim 15$ & 5 & 4 & $25 \sim 35$ & 25 & 6 & $70 \sim 91$ & 100 \\
\hline
\end{tabular}

\subsection{Calculation Method of Network Characteristics}

The degree of a node refers to the number of sides connected to the node. Betweenness:

$$
\mathrm{C}_{\mathrm{B}}(\mathrm{v})=\sum_{\mathrm{s} / \mathrm{v} / \mathrm{t} \in \mathrm{V}} \frac{\sigma_{\mathrm{st}}(\mathrm{v})}{\sigma_{\mathrm{st}}}
$$

$\sigma_{s t}(v)$ represents the shortest path of $\mathrm{s} \rightarrow t$ passing through node $v . \sigma_{s t}$ is the shortest path number for $s \rightarrow t$. Centralization:

$$
C(G)=\sum_{v}\left(\max _{w} c_{w}-c_{v}\right)
$$

where $c_{v}$ is the central value of node $v$ and $C(G)$ is the central value of the network relationship at the graph level.

\subsection{Summary of Software and Analysis Process}

Data were analyzed and figures were drawn using the vegan [15], psych [16], igraph [17], and pheatmap [18] packages in R software [19] and ArcGIS 10.2. The obtained data were standardized according to the indicators; then, the biodiversity indexes and samples (nature reserves) were analyzed by cluster analysis, and the analysis results are shown by heat map and network map. The categories of biodiversity and samples were obtained, and the characteristics of the categories were summarized. Based on the corresponding analysis of environmental factor data and biodiversity data, the main components of environmental factors and the components of influencing biodiversity factors were 
obtained. Taking the nature reserves as the ecological source, the minimum cost path module in GIS was used to analyze the potential corridor of biodiversity diffusion between the source areas. The network structure of the potential corridors was analyzed, the nature reserves and biodiversity diffusion corridors were classified, and their characteristics were summarized.

\section{Results}

\subsection{Relationship between Nature Reserves and Biodiversity}

\subsubsection{Classification of Biodiversity Indicators and Nature Reserves}

The Euclidean method was used for the distance between indicators and nature reserves, and the average was used to determine the clustering. By exploring different quantities and methods of classification, the biodiversity indicators and nature reserves were divided into nine categories (Figure 1, Table 3).

Table 3. Results of classification of biodiversity indicators.

\begin{tabular}{|c|c|}
\hline Category & Biodiversity Indicators \\
\hline First & $\begin{array}{l}\text { number of evergreen broad-leaved forest type (V59), number of evergreen deciduous } \\
\text { broad-leaved mixed forests type (V60), number of mossy dwarf forests type (V62), number of } \\
\text { coniferous forest type (V65) }\end{array}$ \\
\hline Second & $\begin{array}{l}\text { number of total species (T1), number of seed plants species (P2), number of seed plant genera } \\
\text { (P3), number of gymnosperm species (P5), number of gymnosperm genera (P6), number of } \\
\text { gymnosperm families (P7), number of angiosperm species (P8), number of angiosperm genera } \\
\text { (P9), number of Rosaceae species (P11), number of Compositae species (P13), number of } \\
\text { Compositae genera (P14), number of Gramineae species (P15), number of Gramineae genera } \\
\text { (P16), number of Orchidaceae species (P17), number of Orchidaceae families (P18), number of } \\
\text { Labiatae species (P19), number of Labiatae genera (P20), number of Polygonaceae species } \\
\text { (P21), number of Magnoliaceae species (P23), number of Magnoliaceae families (P24), number } \\
\text { of Hepaticae species (P28), number of fern species (P34), number of animal species (A37), } \\
\text { number of mammal species (A39), number of reptile species (A44), number of insect species } \\
\text { (A53), number of insect families (A54), number of insect orders (A55) }\end{array}$ \\
\hline Third & number of reptile orders (A46) \\
\hline Fourth & $\begin{array}{c}\text { number of seed plant families (P4), number of Rosaceae genera (P12), number of Polygonaceae } \\
\text { genera (P22), number of spore plant species (P25), number of spore plant genera (P26), number } \\
\text { of spore plant families (P27), number of Hepaticae genera (P29), number of Hepaticae families } \\
\text { (P30), number of moss species (P31), number of moss genera (P32), number of moss families } \\
\text { (P33), number of fern genera (P35), number of fern families (P36), number of mammal species } \\
\text { (A38), number of orders (A40), number of Aves species (A41), number of Aves families (A42), } \\
\text { number of Aves orders (A43), number of reptile families (A45), number of amphibian species } \\
\text { (A47), number of amphibian families (A48), number of fish species (A50), number of fish } \\
\text { families (A51), number of fish orders (A52) }\end{array}$ \\
\hline Fifth & number of bamboo forest type (V58) \\
\hline Sixth & $\begin{array}{l}\text { number of shrubs type (V57), number of coniferous and broad-leaved mixed forest type (V64), } \\
\text { number of agricultural vegetation type (V67) }\end{array}$ \\
\hline Seventh & number of angiosperm families (P10) \\
\hline Eighth & $\begin{array}{c}\text { number of amphibian orders (A49), number of grassland type (V56), number of deciduous } \\
\text { broad-leaved forest type (V61) }\end{array}$ \\
\hline Ninth & $\begin{array}{l}\text { number of shrub forests type (V63), number of economic forest type (V66), number of moist } \\
\text { meadow type (V68), number of aquatic vegetation type (V69), number of swamp type (V70) }\end{array}$ \\
\hline
\end{tabular}

The first category of biodiversity indicators represents the distribution of vegetation from south to north or vertically and symbolizes the diversity of the ancient components of biological origin. 
The second category mainly represents the number of species and the number of genera and mainly characterizes the biodiversity of plants and small animals at the species level.

The third category includes animals that usually do not migrate, number of reptile orders (A46), which has significant importance to animal geography [20], and characterizes the origin of the original biodiversity.

The fourth category includes many indicators of animal species diversity. The indicators of plant diversity are often the number of genera and families, which characterize the diversity of middle- to large-sized animal species and plant families.

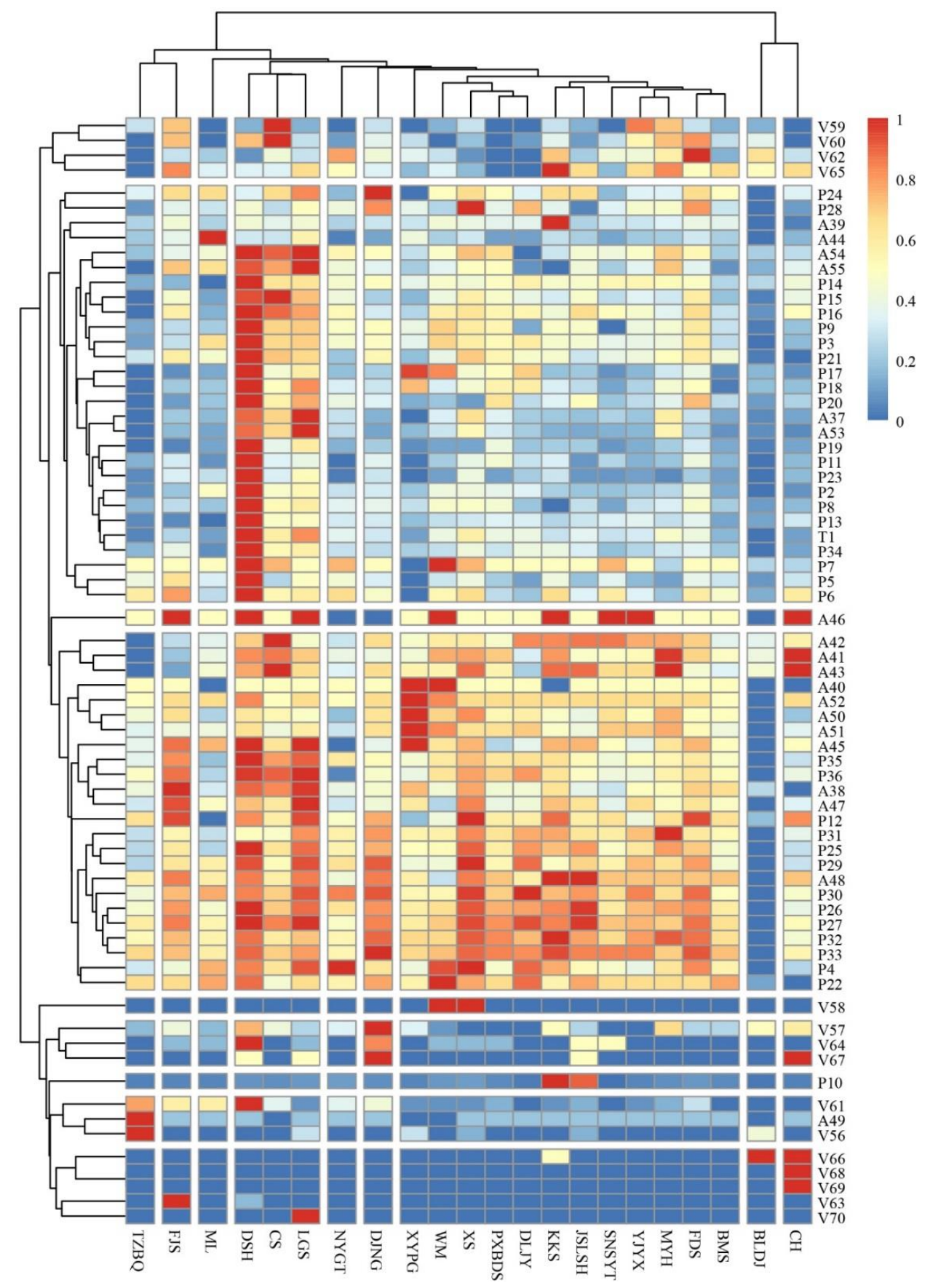

Figure 1. Similarity in the relationship between biodiversity and diversity indicators in nature reserves.

The fifth category is the number of shrub forests (V58). Guizhou is in the subtropical zone and is warm and humid in spring and summer. The zonal climax vegetation is arbor forest, and the 
shrub forests have been naturally restored by secondary succession, which characterizes the temporal diversity of the different vegetation types.

The sixth category includes the number of shrubs (V57), coniferous and broad-leaved mixed forest (V64), and agricultural vegetation (V67). Shrub and agricultural vegetation represent ecosystems that have been impacted by human activities and the transition from a natural ecosystem to human civilization. The coniferous and broad-leaved mixed forests are transitional types of vegetation that are often distributed in belts. This category characterizes the spatial diversity of transitional vegetation ecosystems.

The seventh category, the number of angiosperm families (P10), characterizes the diversity of plant types with close phylogenetic origins.

The eighth category includes the number of amphibian orders (A49), grassland (V56), and evergreen deciduous broad-leaved mixed forests (V61). As Guizhou Province is mainly represented by a zonal climax vegetation community, grassland ecosystems are mainly formed by human activities. The evergreen deciduous broad-leaved forest represents zonal vegetation, while amphibians represent the diversity of native ecosystems in the water environment. This category characterizes the ecosystem diversity over a large region.

The ninth category characterizes the diversity of vegetation types in small regions.

For nature reserves, the first category, which includes the Tongzi Baiqing Nature Reserve (TZBQ), is characterized by moderate biodiversity, many types of grassland vegetation, few birds, and a single type of coniferous forest.

The second category, which is represented by the Fanjingshan Nature Reserve (FJS), has the characteristics of high reptile species richness, unique mossy dwarf forest vegetation types, and gymnosperm dominance.

The third category, the Maolan Nature Reserve (ML), has a single type of vegetation and shows high diversity in plant genera and families, indicating that it is characterized by higher phylogenetic diversity than that in other areas.

The fourth category, which includes the Dashahe Nature Reserve (DSH), Chishui Nature Reserve (CS), and Leigongshan Nature Reserve (LGS), has high animal and plant species richness and numerous vegetation types.

The fifth category is represented by the Nayong Gongtong Nature Reserve (NYGT). This reserve is mainly characterized by high secondary vegetation diversity and high interference intensity.

The sixth category, the Dejiang Nangan Nature Reserve (DJNG), is mainly characterized by higher phylogenetic diversity than that in other protected areas.

The seventh category, including the Xingyi Pogang (XYPG), Wangmo (WM), Xishui (XS), Panxian Badashan (PXBDS), Duliujiangyuan (DLJY), Kuangkuoshui (KKS), Jinsha Lengshuihe (JSLSH), Sinan Siyetun (SNSYT), Yinjiang Yangxi (YJYX), Mayanghe (MYH), Fodingshan (FDS), and Baimianshui (BMS) nature reserves, has intermediate and high biodiversity, relatively rich coniferous forest types, and an intermediate number of animal species, especially insect species. However, characteristic vegetation types are not prominent, which is common type of biodiversity.

The eighth category, the Bailidujuan Nature Reserve (BLDJ), has high numbers of shrub species (mainly species of Rhododendron), grasses, and economic forest vegetation types, a single type of forest vegetation, and low biodiversity.

The ninth category, the Caohai Nature Reserve $(\mathrm{CH})$, has characteristic aquatic vegetation, wet meadows, economic forests, and agricultural vegetation types. This reserve has extremely high bird species richness and has no forest vegetation, and it has the characteristics of alternative biodiversity.

\subsubsection{Network Relationships of the Nature Reserves}

Correlation coefficients were calculated based on the biodiversity data for each nature reserve, and the correlation coefficients $\geq 0.6$ with $p$ values $<0.01$ were selected. The community structure of each nature reserve was assessed via greedy optimization of modularity [21]. The network relationship 
analysis shows (Figure 2) that the 22 nature reserves were divided into seven communities (modularity value $=0.134)$. Four nature reserves, Caohai $(\mathrm{CH})$, Maolan $(\mathrm{ML})$, Bailidujuan (BLDJ), and Tongzi Baiqing (TZBQ), were considered independent categories because their biodiversity characteristics differed from those of the other nature reserves. Seven nature reserves, Xingyi Pogang (XYPG), Wangmo (WM), Duliujiangyuan (DLJY), Dashahe (DSH), Panxian Badashan (PXBDS), Xishui (XS), and Leigongshan (LGS), composed one community, of which DLJY, PXBDS, and XS were the main nodes connected with the other communities. Four nature reserves, Fodingshan (FDS), Mayanghe (MYH), Nayong Gongtong (NYGT), and Chishui (CS), formed another group. FDS and MYH were the main nodes that were connected with the other types of nature reserves. Seven nature reserves, Dejiang Nangan (DJNG), Baimianshui (BMS), Sinan Siyetun (SNSYT), Jinsha Lengshuihe (JSLSH), Yinjiang Yangxi (YJYX), Fanjingshan (FJS), and Kuangkuoshui (KKS), also formed a group; SNSYT, BMS, and YJYX were the main nodes that were connected to the other types of nature reserves.

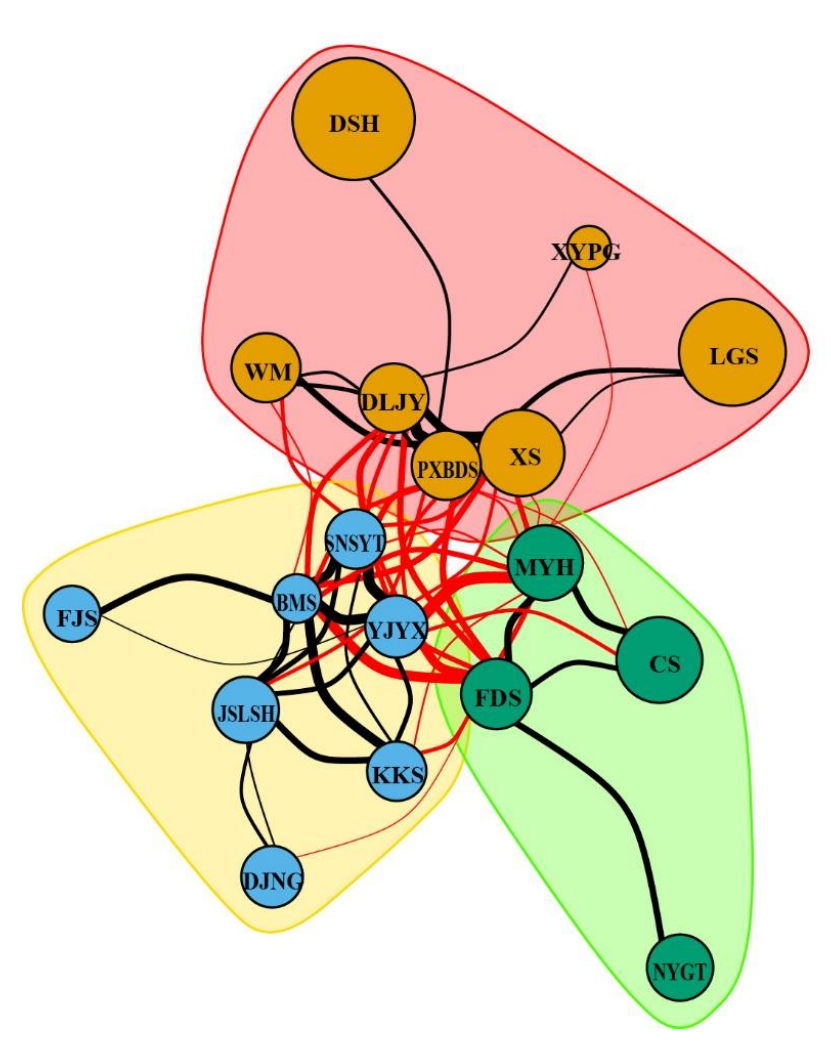

Figure 2. Network analysis of biodiversity in nature reserves of Guizhou Province. The width of the connecting line is related to the correlation coefficient $(p \leq 0.01)$ of the biodiversity of the protection interval. The circles in the figure indicate the protected areas. The size of the circle is positively correlated with the total number of species in the protected area. The color of the circle indicates its group. Nature reserve name abbreviations: TZBQ: Tongzi Baiqing, FJS: Fanjingshan, ML: Maolan, DSH: Dashahe, CS: Chishui, LGS: Leigongshan, NYGT: Nayong, DJNG: Dejiang Nengan, XYPG: Xingyi Piang, WM: Wangmo, XS: Xishui, PXBDS: Panxian Badashan, DLJY: Duliujiangyuan, KKS: Kuankuoshui, JSLSH: Jinsha Ligshuihe, SNSYT: Sinan Situn, YJYX: Yinjiang Yiangxi, MYH: Mayanghe, FDS: Fodingshan, BMS: Bannshui, BLDJ: Bailidujuan, and CH: Caohai. 
At the graph level, the network centralization score of betweenness was 0.121 , and centralization score of degree was 0.325 , which indicated that the centrality was low, and the connectivity was at an intermediate level. The network characteristics of the biodiversity relationships are shown in Table 4. The main nodes connected to the other communities had high centralization scores of betweenness and degree; they were key nodes in the network and showed good connection and transition in the corresponding categories and the other categories. According to the node size (biological species richness), these nodes were at a medium level, which may be the reason that they were key nodes in the network.

Table 4. Network relationship characteristics of biodiversity correlation in the nature reserves of Guizhou Province.

\begin{tabular}{|c|c|c|c|c|}
\hline Abbreviation & Nature Reserve & Community & Betweenness & Degree \\
\hline BMS & Baimianshui & 1 & 12.61 & 12 \\
\hline SNSYT & Sinan Siyetun & 1 & 5.51 & 11 \\
\hline YJYX & Yinjiang Yangxi & 1 & 2.89 & 9 \\
\hline JSLSH & $\begin{array}{l}\text { Jinsha } \\
\text { Lengshuihe }\end{array}$ & 1 & 2.80 & 9 \\
\hline DJNG & Dejiang Nangang & 1 & 0.00 & 6 \\
\hline KKS & Kuangkuoshui & 1 & 0.00 & 4 \\
\hline FJS & Fanjingshan & 1 & 0.00 & 2 \\
\hline PXBDS & $\begin{array}{l}\text { Panxian } \\
\text { Badashan }\end{array}$ & 2 & 40.35 & 15 \\
\hline FDS & Fodingshan & 2 & 20.85 & 13 \\
\hline XS & Xishui & 2 & 4.02 & 10 \\
\hline MYH & Mayanghe & 2 & 1.61 & 7 \\
\hline CS & Chishui & 2 & 0.00 & 3 \\
\hline LGS & Leigongshan & 2 & 0.00 & 3 \\
\hline NYGT & $\begin{array}{l}\text { Nayong } \\
\text { Gongtong }\end{array}$ & 2 & 0.00 & 2 \\
\hline $\mathrm{DSH}$ & Dashahe & 2 & 0.00 & 1 \\
\hline DLJY & Duliujiangyuan & 3 & 4.98 & 10 \\
\hline WM & Wangmo & 3 & 1.40 & 6 \\
\hline XYPG & Xingyi Pogang & 3 & 0.00 & 3 \\
\hline TZBQ & Tongzi Baiqing & 4 & 0.00 & 0 \\
\hline BLDJ & Bailidujuan & 5 & 0.00 & 0 \\
\hline ML & Maolan & 6 & 0.00 & 0 \\
\hline $\mathrm{CH}$ & Caohai & 7 & 0.00 & 0 \\
\hline
\end{tabular}

\subsubsection{CCA of Biodiversity and Ecological Factors in Nature Reserves}

In this study, 36 environmental factors were selected for the canonical correspondence analysis of the biodiversity of 22 nature reserves in Guizhou Province. The total interpretation rate of the 36 environmental factors for biodiversity variation reached 100\%. The CCA axis features show that the first two axes explain $41.4 \%$ of the biodiversity variation (Figure 3 ).

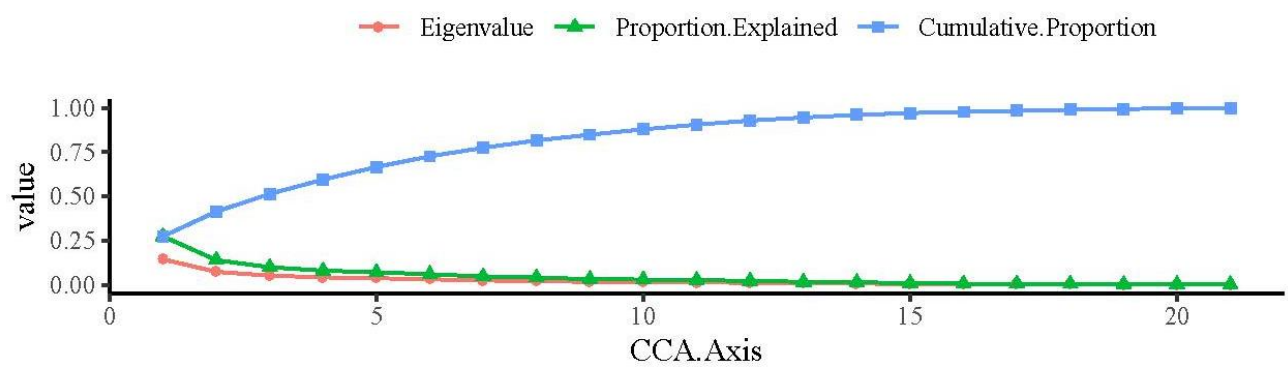

Figure 3. Axis eigenvalue and interpretation of the rate distribution of the canonical correspondence analysis (CCA). 
The CCA plot (Figure 4) shows that the differences in environmental factors among the nature reserves (the length of the arrows) are explained by the minimum altitude (E89), followed by the coal-bearing stratum (R45), marsh soil (S11), lithology (R78), north temperate distribution (G100), quartz sandstone (R52), relative height difference (E90), annual precipitation (C92), discontinuous distribution in tropical Asia and tropical America (G95), and annual average temperature (C91).

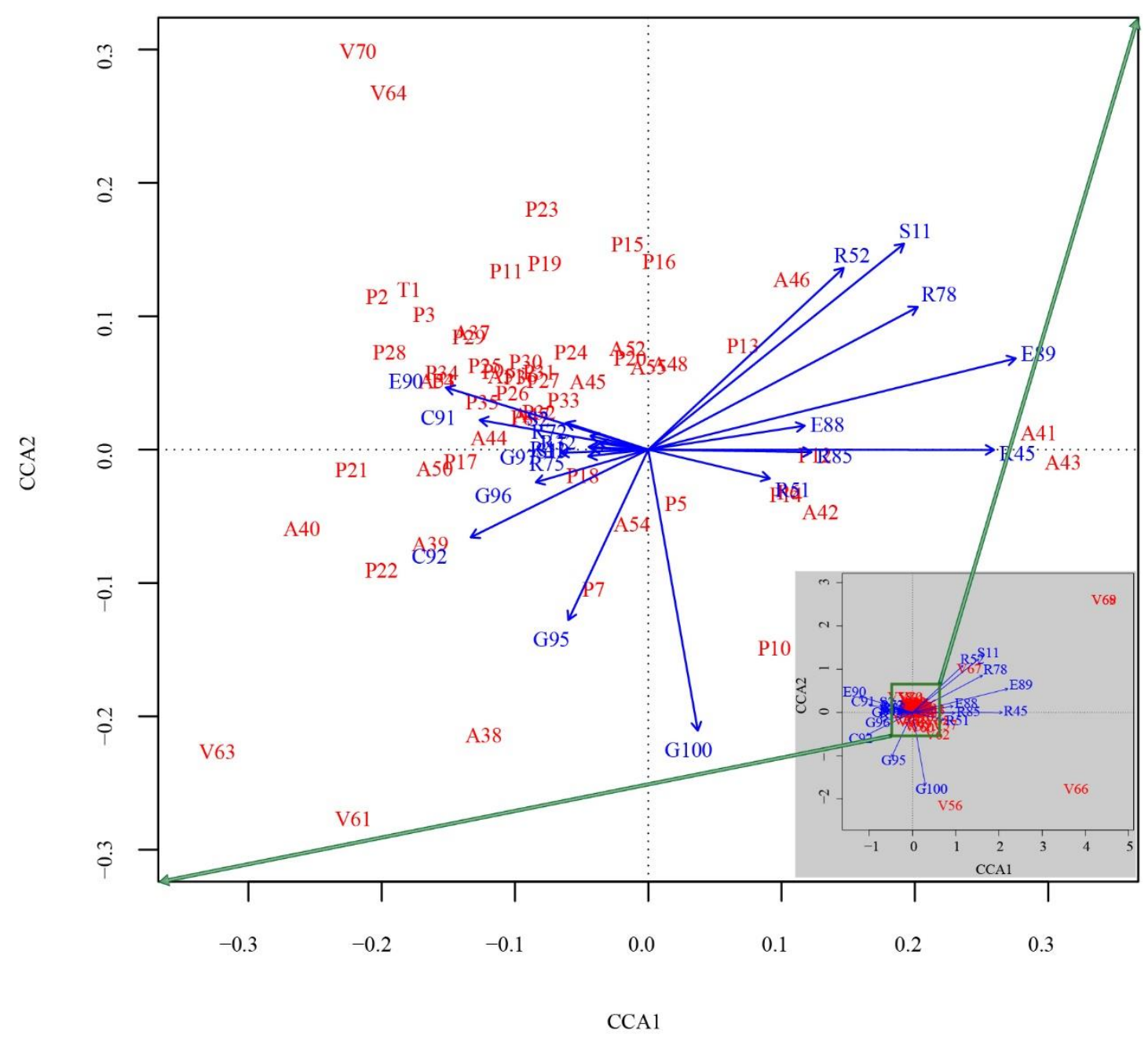

Figure 4. CCA of biodiversity and ecological factors in Guizhou Province.

\subsubsection{Ecological Significance of the CCA Coordinate Axis}

The relationships between environmental factors and the CCA axes were examined by changing the permutation test 9999 times. A total of 25 ecological factors with large correlation coefficients $(\geq 0.95)$ or significant impact on biodiversity $(p \leq 0.10)$ were identified (Table 5$)$. The ecological factors that showed a very significant effect on biodiversity $(p<0.01)$ were the minimum altitude and coal stratum; those that had a significant impact on biodiversity $(p<0.05)$ were the relative height difference, quartz sandstone, marsh soil, and proportion of plants with a north temperate distribution. The factors that had a great impact on biodiversity $(p \leq 0.10)$ were fuchsia shale, the proportion of plants with a discontinuous distribution in tropical Asia and tropical America, yellow sand shale, and annual precipitation. 
Table 5. The most important ecological factors and their relationships with the environmental axes obtained from the CCA.

\begin{tabular}{|c|c|c|c|c|c|c|}
\hline Type & Abbreviation & Ecological Factor & CCA1 & CCA2 & $r^{2}$ & $p$ \\
\hline \multirow[t]{3}{*}{ Terrain } & E89 & Minimum altitude & 0.971 & 0.241 & 0.568 & $0.01^{* *}$ \\
\hline & E90 & Relative height difference & -0.956 & 0.293 & 0.173 & 0.04 * \\
\hline & E88 & Highest altitude & 0.989 & 0.151 & 0.100 & 0.20 \\
\hline \multirow[t]{2}{*}{ Climate } & $\mathrm{C} 92$ & Annual precipitation & -0.897 & -0.443 & 0.156 & 0.10 \\
\hline & C91 & Annual average temperature & -0.985 & 0.173 & 0.117 & 0.13 \\
\hline \multirow[t]{2}{*}{ Soil } & S11 & Marsh soil & 0.779 & 0.627 & 0.429 & $0.04 *$ \\
\hline & S17 & Coarse soil & -0.995 & 0.102 & 0.010 & 0.90 \\
\hline \multirow[t]{15}{*}{ Lithology } & R45 & Coal stratum & 1.000 & -0.001 & 0.474 & $0.01 * *$ \\
\hline & $\mathrm{R} 52$ & Quartz sandstone & 0.733 & 0.681 & 0.284 & 0.04 * \\
\hline & R78 & Fuchsia shale & 0.884 & 0.468 & 0.369 & 0.07 \\
\hline & R87 & Yellow sand shale & 0.560 & -0.828 & 0.803 & 0.09 \\
\hline & R73 & Silty shale & 0.189 & -0.982 & 0.026 & 0.30 \\
\hline & $\mathrm{R} 51$ & Limestone & 0.973 & -0.231 & 0.061 & 0.40 \\
\hline & R39 & Mud shale & 0.169 & -0.986 & 0.016 & 0.45 \\
\hline & $\mathrm{R} 41$ & Limestone & -0.999 & 0.045 & 0.014 & 0.51 \\
\hline & $\mathrm{R} 72$ & Silty mudstone & -0.973 & 0.232 & 0.014 & 0.52 \\
\hline & $\mathrm{R} 34$ & Mudstone & 0.064 & -0.998 & 0.017 & 0.57 \\
\hline & R75 & Fuchsia mudstone & -0.994 & -0.109 & 0.014 & 0.68 \\
\hline & $\mathrm{R} 32$ & Slate & -0.992 & 0.129 & 0.009 & 0.70 \\
\hline & R59 & Sand shale & 0.276 & -0.961 & 0.017 & 0.91 \\
\hline & $\mathrm{R} 50$ & Dolomitic limestone & 0.237 & -0.971 & 0.006 & 0.94 \\
\hline & R67 & Carbonate & -0.954 & -0.299 & 0.003 & 0.96 \\
\hline $\begin{array}{c}\text { Plant } \\
\text { geographical } \\
\text { composition }\end{array}$ & G100 & North temperate distribution & 0.174 & -0.985 & 0.324 & $0.05^{*}$ \\
\hline
\end{tabular}

The relationships between ecological factors and the CCA axes (Table 5) showed that the first axis consisted of three terrain factors, two climate factors, four soil factors, 10 lithology factors, and three plant geographical composition factors. Among these factors, the terrain, climatic, and plant geographic area factors mainly represented heat and precipitation conditions. Soil factors, including red soil, purple red shale, purple red mudstone, coal stratum, and marsh soil, also refer to heat and are characterized by the presence of water on the terrain, such as lakes and swamps. According to the CCA plot, the ecological significance of the first axis of the CCA represents cold, dry, and low precipitation conditions and deep rock formations. The second axis consists of nine lithology factors and five plant geographical composition ratio factors. The lithology is dominated by metamorphic rocks; they are soil-forming parent rocks that weather and form deep soil. The plant geographical area shows an intermittent distribution and characteristic area, and it is dominated by plants that originate from warm regions. The second axis of the CCA is considered to characterize deep soil.

\subsubsection{Relationships between Environmental Factors and Biodiversity}

The CCA ranking map of biodiversity indicators and environmental factors shows that the types of biodiversity indicators that are affected by environmental factors from obvious to nonsignificant impacts are the number of vegetation types, total number of species, number of seeds, number of plant species of relatively early origin, number of animals species, number of angiosperms, number of spore plants, number of species-rich families, and number of gymnosperms.

The diversity indicators that are significantly affected by environmental factors are wet meadow (V68) and aquatic vegetation (V69) for the wetland vegetation types; the total number of species (P2) 
and genera (P3) for seed plants; the number of Lauraceae species (P21), the number of Lauraceae genera (P22), and the number of Magnolia species (P23) for the early-originating plant species; the number of amphibian orders (A49), Aves orders (A43), Aves species (A41), mammal orders (A40), and mammal species (A38) for animals; the number of angiosperm families (P10) and the numbers of moss (P28) and fern (P34) species for spore plants; the number of Rosaceae species (P11) and lip species (P19) for the species-rich families; and the number of gymnosperm families (P7).

\subsection{Corridors for the Spread of Regional Biodiversity}

The spread and exchange of regional biodiversity will be affected by many factors. All types of ecological factors have certain effects. At the level of specific ecological factors, it is difficult to identify a unified law or theoretical model, but at the macro level, such a model is nothing more than the biological selection of habitats and the barriers/impacts of natural or human activities. Therefore, this study uses the land use type as the basic data for habitat selection, which were supplemented with the effects of human activity interference in the various land use types. Moreover, DEM data are used to calculate the slope of the area, which provides the basic data for natural barriers.

According to the analysis of the law of biodiversity diffusion, the resistance surface of biodiversity diffusion in Guizhou Province was established, and the minimum arrival cost paths for 21 nature reserves in Guizhou Province were analyzed to form a spatial relationship network of ecological corridors for biodiversity diffusion (Figure 5).

Each nature reserve was considered a node, and according to the definition of degree and number, the characteristics of the ecological corridor network relationship of the nature reserves in Guizhou Province were assessed.

The actual meaning of node betweenness is the sum of the number of times that the organisms in each nature reserve pass through a node when passing through the other nodes. The actual meaning of node degree is the number of nature reserves directly connected to the node. Edge betweenness represents a common ecological corridor. In this study, the biodiversity corridors were divided into six levels $(1-4,5-8,9-12,13-16,17-20,21-24)$ based on the median value of the edge. High median levels indicate high betweenness.

The analysis of the network relationship characteristics (Table 6) shows that based on the diffusion and attraction of biodiversity among the nature reserves, a good ecological corridor network for biodiversity diffusion can be created among the nature reserves. According to the characteristics of each nature reserve, the Kuangkuoshui reserve has the highest degree, indicating that this reserve is at the center of the network, and its median value is the highest, indicating that the biodiversity of the protected area mostly spreads through this path.

Table 6. Node characteristics of the spatial network relationships among nature reserves.

\begin{tabular}{cccccc}
\hline Nature Reserve & Note Betweenness & Note Degree & Nature Reserve & Note Betweenness & Note Degree \\
\hline Kuangkuoshui & 42 & 7 & Duliujiangyuan & 15 & 3 \\
Baimianshui & 41 & 6 & Xishui & 5 & 4 \\
Bailidujuan & 37 & 3 & Dashahe & 5 & 3 \\
Jinsha Lengshui & 37 & 5 & Xingyi Pogang & 2 & 3 \\
Nayong Gongtong & 31 & 4 & Maolan & 2 & 1 \\
Fodingshan & 28 & 5 & Caohai & 1 & 2 \\
Dejiang Nangang & 27 & 5 & Chishui & 1 & 2 \\
Yinjiang Yangxi & 25 & 5 & Mayanghe & 1 & 3 \\
Leigongshan & 21 & 2 & Tongzi Baiqing & 0 & 1 \\
Sinan Siyetun & 19 & 5 & Fanjingshan & 0 & \\
Wangmo & 15 & 3 & & & \\
\hline
\end{tabular}

The edge betweenness values of the ecological corridors were accounted for and classified into six levels (level 1: 1-4, level 2: 5-8, level 3: 9-12, level 4: 13-16, level 5: 17-20, level 6: 21-24). The five corridors with the highest values are distributed in three areas. Two of them are corridors in the 
Jinsha Lengshuihe-Baimianshui and Jinsha Lengshuihe-Nayong Gontong nature reserves. Two of them are corridors that connect the Leigongshan-Duliujiangyuan and Maolan nature reserves and the Leigongshan-Fodingshan and Yinjiang Yangxi nature reserves. One is the Nayong Gongtong-Balidujun corridor. The total length of the corridors is $3258.5 \mathrm{~km}$ (Table 7), and the average length is $66 \mathrm{~km}$. The total length of the level-2 corridors is the longest, the total length of the level-3 corridors is the shortest, the average length of the level-5 corridors is the shortest, and the average length of the level-1 corridors is the longest.

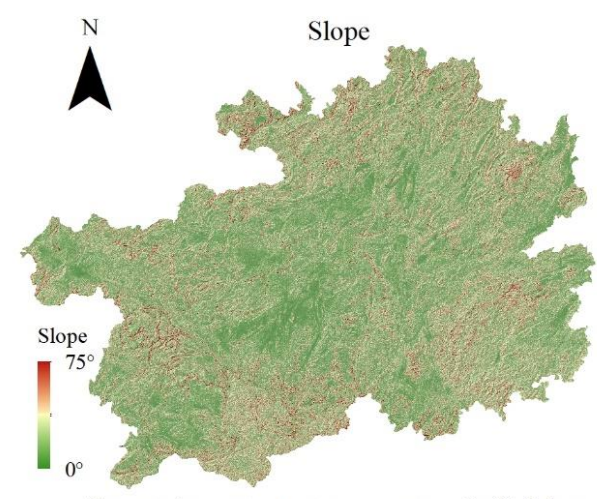

Twenty important nature reserves in Guizhou

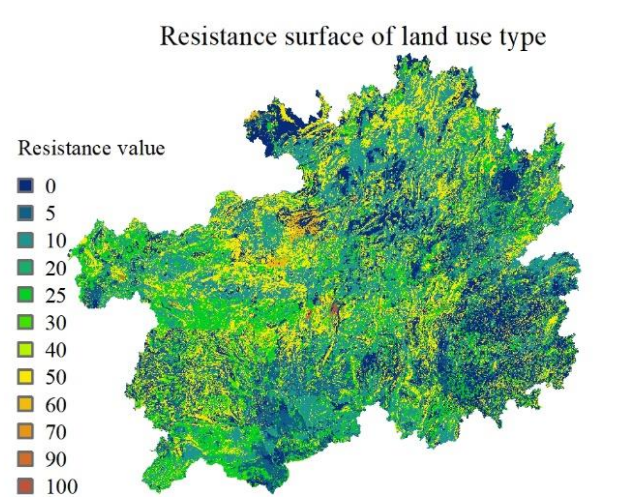

Cost model (total resistance) of biodiversity diffusion

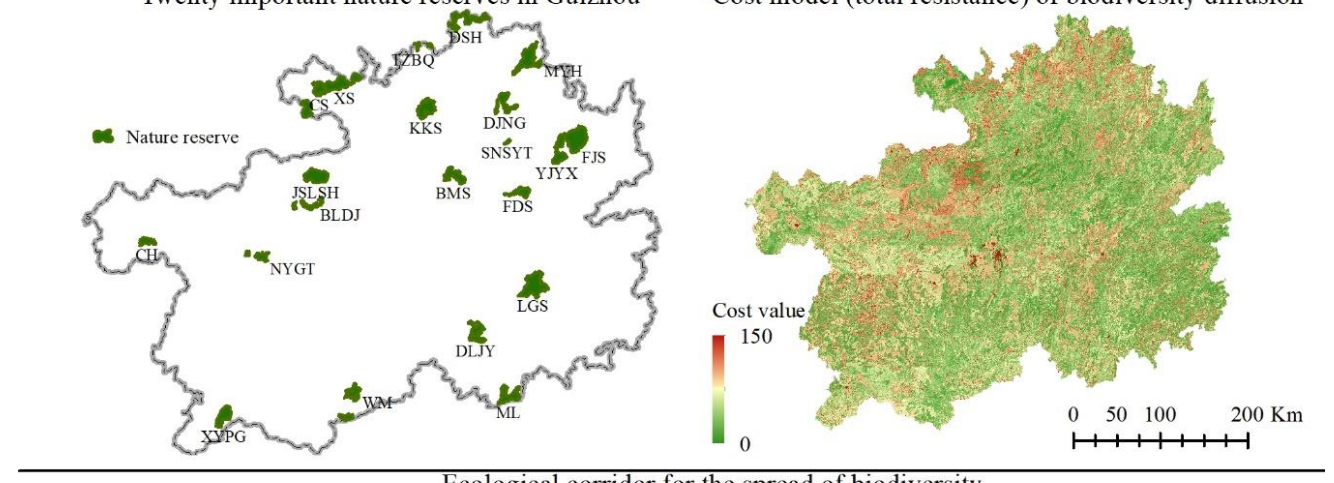

Ecological corridor for the spread of biodiversity

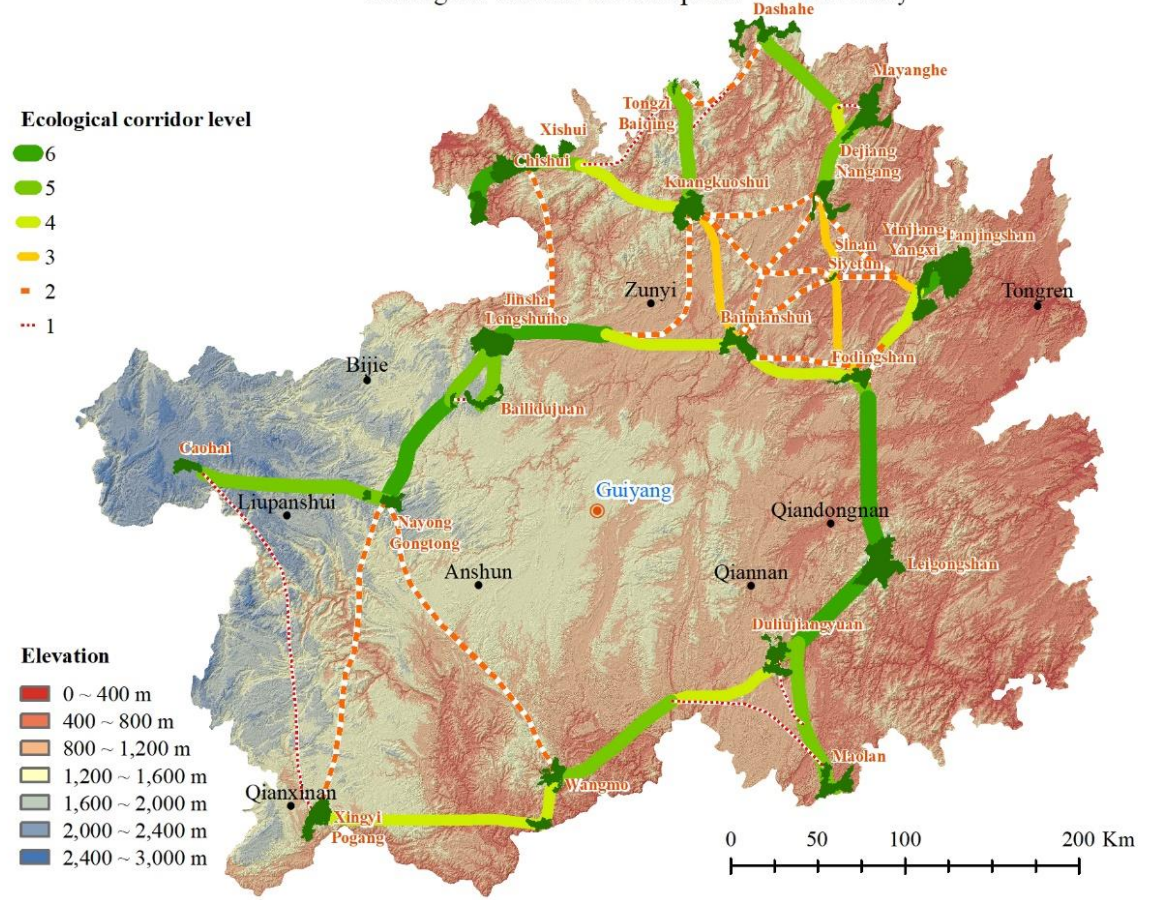

Figure 5. Analysis of ecological corridors for biodiversity diffusion in nature reserves. 
Table 7. Characteristics of ecological corridors for biodiversity diffusion in Guizhou Province.

\begin{tabular}{cccccc}
\hline Corridor Level & Amount & Total Length $/ \mathbf{k m}$ & Length Percentage $/ \%$ & Average Length $/ \mathbf{k m}$ & Area $/ \mathbf{k m}^{\mathbf{2}}$ \\
\hline 6 & 5 & 299.3 & 9.2 & 59.9 & 119.7 \\
5 & 12 & 536.2 & 16.5 & 44.7 & 214.5 \\
4 & 7 & 475.6 & 14.6 & 67.9 & 190.2 \\
3 & 4 & 211.4 & 6.5 & 52.8 & 84.6 \\
2 & 15 & 1228.5 & 37.7 & 81.9 & 491.4 \\
1 & 6 & 507.5 & 15.6 & 84.6 & 203.0 \\
Total & 49 & 3258.5 & 100 & 66.5 & 1303.4 \\
\hline
\end{tabular}

\subsection{Analysis of the Characteristics of Nature Reserves in Guizhou Province}

According to the analysis of biodiversity and spatial transmission path characteristics, the nature reserves in Guizhou Province can be categorized based on their biodiversity status and the locations of the biodiversity diffusion corridors (Table 8).

Table 8. Comprehensive characteristics of nature reserves in Guizhou Province.

\begin{tabular}{lll}
\hline No. & Feature Category & Nature Reserves \\
\hline 1 & High-biodiversity reserve & Dashahe, Fanjingshan, Chishui \\
2 & Secondary node nature reserve with high biodiversity & Xishui, Mayanghe \\
3 & Secondary regional center nature reserve with high biodiversity & Leigongshan \\
4 & Distinctive regional center nature reserve & Bailidujuan \\
5 & Distinctive nature reserve & Tongzi Baiqing, Maolan, Caohai \\
6 & Regional nature reserve & Xingyi Pogang \\
7 & Secondary regional center nature reserve with regional characteristics & Wangmo, Duliujiangyuan \\
8 & Medium- and high-biodiversity important node nature reserve & Panxian Badashan \\
9 & Medium- and high-biodiversity secondary regional center important node nature reserve & Fodingshan \\
10 & Medium- and high-biodiversity secondary regional center nature reserve & Nayonggongtong \\
11 & Secondary biosphere diversity regional center secondary node nature reserve & Jinsha Lengshuihe \\
12 & Important node nature reserve of the central biodiversity special important regional center & Baimianshui \\
13 & Medium-biodiversity special important regional center nature reserve & Kuangkuoshui \\
14 & Medium-biodiversity secondary regional center nature reserve & Dejiang Nangang \\
15 & Secondary biodiversity secondary regional center secondary node nature reserve & Yinjiang Yangxi, Sinan Siyetun \\
\hline
\end{tabular}

\section{Conclusions and Discussion}

According to the correlations of biodiversity in Guizhou Province, the characteristics of the spatial location network, and the analysis of the biodiversity diffusion corridors, the following meaningful conclusions can be draw.

When the classification of similar biodiversity groups in nature reserves is combined with the environmental factor/nature reserve/biodiversity CCA, the nature reserves of Guizhou Province can be classified into seven categories.

The ecological factors that mainly affect regional biodiversity are heat, moisture, rock type, maternal rock type, and soil type, indicating that the ecological factors affecting biodiversity are complex and diverse.

The total length of the ecological biodiversity diffusion corridors in Guizhou Province calculated in this study is $3258.5 \mathrm{~km}$, and the corridors are divided into levels of importance. Government departments can implement ecological construction measures for important corridors, create protected areas in low-level corridor areas, increase the number of network nodes, and develop more biodiversity and ecological sources and channels. The construction of forest corridors with a width of $400 \mathrm{~m}$ can support the diffusion and survival of most species [22]. According to this value (Table 6), the total area of ecological corridors that should be built in Guizhou Province for biodiversity diffusion is $1303.4 \mathrm{~km}^{2}$, accounting for $0.74 \%$ of the total area of Guizhou Province $\left(176,176 \mathrm{~km}^{2}\right)$.

It is almost impossible and unnecessary to build such a wide corridor in densely populated areas. However, if the ecological corridor bypasses the edge of urban planning, the people in the area concede part of the agricultural land, build some corridor bridges across the vehicle road, and do 
some protection measures to the ecological corridor, the possibility of making a $400 \mathrm{~m}$ wide ecological corridor network is still relatively high.

In this study, some explorations were carried out on the pattern, index relationship, spatial relationship, and node function of biodiversity, the influencing factors of regional biodiversity were put forward, and the measures to improve the spatial pattern of biodiversity islands were evaluated. However, there are still many shortcomings. Future studies should be based on a deeper quantitative assessment of biological phylogenetic diversity and population size. In the interpretation of spatial location and formation mechanism, it is necessary to determine the parameters of environmental factors. Larger regional studies can be carried out to verify these conclusions. Problems such as the fact that the timing of macro data are out-of-sync and the depth of data acquisition is inconsistent may be the next steps that need to be paid attention to. The biodiversity data and analysis with time series should also be paid more attention to.

Author Contributions: Conceptualization, L.Y. and M.A.; methodology, L.Y.; software, L.Y.; validation, L.Y., M.A., and H.S.; formal analysis, L.Y.; investigation, L.Y., H.L., and C.Y.; resources, L.Y., H.L., and C.Y.; data curation, L.Y.; writing — original draft preparation, L.Y.; writing — review and editing, L.Y. and M.A.; visualization, L.Y.; supervision, L.Y. and M.A.; project administration, L.Y. and M.A.; funding acquisition, L.Y.

Funding: This study was funded by The Project of National Key Research and Development Program of China (grant number 2016YFC0502604), the Major Project of Guizhou Province (Qian Ke He Major Project [2016]3022), the Special Project on Biodiversity Conservation of the Ministry of Environmental Protection (grant number kksbdyj01), the Construction Program of Biology First-class Discipline in Guizhou (grant number GNYL[2017]009), and the Project of Promoted Innovation for Colleges and Universities of Guizhou Province (grant number Qian Jiao He Collaborative Innovation [2014]01).

Conflicts of Interest: The authors declare no conflict of interest.

\section{References}

1. Fischer, J.; Lindenmayer, D.B. Landscape modification and habitat fragmentation: A synthesis. Glob. Ecol. Biogeogr. 2007, 16, 265-280. [CrossRef]

2. Liu, Q.; Yang, Z.; Wang, F. Conservation Policy-Community Conflicts: A Case Study from Bogda Nature Reserve, China. Sustainability 2017, 9, 1291. [CrossRef]

3. Yang, H.; Harrison, R.; Yi, Z.; Goodale, E.; Zhao, M.; Xu, J. Changing perceptions of forest value and attitudes toward management of a recently established nature reserve: A case study in southwest China. Forests 2015, 6, 3136-3164. [CrossRef]

4. Laurance, W.F.; Clements, G.R.; Sloan, S.; O'Connell, C.S.; Mueller, N.D.; Goosem, M.; Venter, O.; Edwards, D.P.; Phalan, B.; Balmford, A.; et al. A global strategy for road building. Nature 2014, 513, $229-232$. [CrossRef] [PubMed]

5. Haddad, N.M.; Brudvig, L.A.; Clobert, J.; Davies, K.F.; Gonzalez, A.; Holt, R.D.; Lovejoy, T.E.; Sexton, J.O.; Austin, M.P.; Collins, C.D.; et al. Habitat fragmentation and its lasting impact on Earth's ecosystems. Sci. Adv. 2015, 1, e1500052. [CrossRef] [PubMed]

6. Wilson, M.C.; Chen, X.; Corlett, R.T.; Didham, R.K.; Ding, P.; Holt, R.D.; Holyoak, M.; Hu, G.; Hughes, A.C.; Jiang, L.; et al. Erratum to: Habitat fragmentation and biodiversity conservation: Key findings and future challenges. Landsc. Ecol. 2016, 31, 229-230. [CrossRef]

7. Ma, K. Frontiers in biodiversity science: Insular biogeography, community assembly and application of big data. Biodivers. Sci. 2017, 25, 343-344. [CrossRef]

8. Patiño, J.; Whittaker, R.J.; Borges, P.A.V.; Fernández-Palacios, J.M.; Ah-Peng, C.; Araújo, M.B.; Ávila, S.P.; Cardoso, P.; Cornuault, J.; de Boer, E.J.; et al. A roadmap for island biology: 50 fundamental questions after 50 years of The Theory of Island Biogeography. J. Biogeogr. 2017, 44, 963-983. [CrossRef]

9. Zhao, S.; Fang, J.; Lei, G. Theoretical basis $\mathrm{f}$ or species conservation: From the theory of islandbiogeography to metapopulation dynamic theory. Acta Ecol. Sin. 2001, 21, 1171-1179. [CrossRef]

10. Wu, J.; Liu, Z. Effect of habitat fragmentation on biodiversity: A review. Chin. J. Ecol. 2014, 33, 1946-1952.

11. Zhuge, H.; Lin, D.; Li, X. Identification of ecological corridors for Tibetan antelope and assessment of their human dis-turbances in the alpine desert of Qinghai-Tibet Plateau. Chin. J. Appl. Ecol. 2015, 26, 2504-2510. 
12. Zhou, Y.; Xu, W.; Zhao, C.; Shen, G.; Xiong, G.; Fan, D.; Xie, Z. Feasibility and technical design for enhancing habitat connectivity across two separatedcomponents in Shennongjia World Natural Heritage Site, China. Chin. J. Ecol. 2017, 36, 2988-2996.

13. Legendre, P.; Legendre, L. Numerical Ecology, 3rd ed.; Elsevier: London, UK, 2012; Volume 24, pp. $625-710$.

14. Borcard, D.; Gillet, F.; Legendre, P. Numerical Ecology with R, 2nd ed.; Springer: Basel, Switzerland, 2018.

15. Oksanen, J.; Blanchet, F.G.; Friendly, M.; Kindt, R.; Legendre, P.; McGlinn, D.; Minchin, P.R.; O'Hara, R.B.; Simpson, G.L.; Solymos, P.; et al. Vegan: Community Ecology Package; CRAN: Vienna, Austria, 2018.

16. Revelle, W. Psych: Procedures for Psychological, Psychometric, and Personality Research; Northwestern University: Evanston, IL, USA, 2018.

17. Csardi, G.; Nepusz, T. The igraph software package for complex network research. Interjournal Complex Syst. 2006, 1695, 1-9.

18. Kolde, R. Pheatmap: Pretty Heatmaps; CRAN: Vienna, Austria, 2018.

19. Team, R.C. R: A Language and Environment for Statistical Computing; R Core Team: Vienna, Austria, 2018.

20. Qu, W.; Lu, J.; Chen, X.; Niu, H.; Lu, J.; Niu, Y. Geographical Division of Reptile in Henan Province. Sichuan J. Zool. 2002, 21, 142-146. [CrossRef]

21. Clauset, A.; Newman, M.E.J.; Moore, C. Finding community structure in very large networks. Phys. Rev. E 2005, 70, 66111. [CrossRef] [PubMed]

22. Kang, M. The Adopt Width of Forest Corridor for Different Species Biodiversity Service. For. Environ. Sci. 2018, 34, 42-46. [CrossRef]

(C) 2019 by the authors. Licensee MDPI, Basel, Switzerland. This article is an open access article distributed under the terms and conditions of the Creative Commons Attribution (CC BY) license (http://creativecommons.org/licenses/by/4.0/). 